\title{
A class of mixed orthogonal arrays obtained from projection matrix inequalities
}

\author{
Shanqi Pang ${ }^{*}$, Yan Zhu and Yajuan Wang
}

${ }^{*}$ Correspondence: pangshanqi@263.net Henan Engineering Laboratory for Big Data Statistical Analysis and Optimal Control, School of Mathematics and Information Science, Henan Normal University, Xinxiang, 453007, China

\begin{abstract}
By using the relationship between orthogonal arrays and decompositions of projection matrices and projection matrix inequalities, we present a method for constructing a class of new orthogonal arrays which have higher percent saturations. As an application of the method, many new mixed-level orthogonal arrays of run sizes 108 and 144 are constructed.
\end{abstract}

MSC: 62K15; 05B15

Keywords: matrix inequality; orthogonal array; orthogonal projection matrix; matrix image

\section{Introduction}

An $n \times m$ matrix $A$, having $k_{i}$ columns with $p_{i}$ levels, $i=1,2, \ldots, r, m=\sum_{i=1}^{r} k_{i}, p_{i} \neq p_{j}$ for $i \neq j$, is called an orthogonal array (OA) of strength $d$ and size $n$ if each $n \times d$ submatrix of $A$ contains all possible $1 \times d$ row vectors with the same frequency. Unless stated otherwise, we use the notation $L_{n}\left(p_{1}^{k_{1}} \cdots p_{r}^{k_{r}}\right)$ for an OA of strength 2 . An orthogonal array is said to have mixed levels if $r \geq 2$. Orthogonal arrays have been used extensively in statistical design of experiments, computer science and cryptography. Constructions of OAs have been studied extensively in the literature; see Hedayet et al. [1, 2], Zhang et al. [3, 7], Pang et al. [5], Pang [6], Zhang et al. [7], Chen et al. [8], Du et al. [9], etc. Zhang et al. [3] present a method of construction of orthogonal arrays of strength two by using a relationship between orthogonal arrays and decompositions of projection matrices. In the construction of new mixed orthogonal arrays, two goals should be kept in mind, first, we want the orthogonal array to be as close to a saturated main-effect plan as possible so that there will be a large number of factors and second, we want the $p_{i}$, the number of levels, to be as large as possible so that the design has a high degree of flexibility (see Mandeli [10]). In this paper by using projection matrix inequalities and further exploring the relationship, matrix images of a class of orthogonal arrays can be found. Therefore we construct a class of new orthogonal arrays. If, as in Mandeli [10], we still define the percent saturation of an OA $L_{n}\left(p_{1}^{k_{1}} \cdots p_{r}^{k_{r}}\right)$ to be $\sum_{i=1}^{r} k_{i}\left(p_{i}-1\right) /(n-1) \times 100 \%$, then the orthogonal arrays constructed in this paper have higher percent saturations.

\section{Basic concepts and main theorems}

The following definitions, notations, and results are needed in the sequel.

(c) 2015 Pang et al. This article is distributed under the terms of the Creative Commons Attribution 4.0 International License (http://creativecommons.org/licenses/by/4.0/), which permits unrestricted use, distribution, and reproduction in any medium, provided you give appropriate credit to the original author(s) and the source, provide a link to the Creative Commons license, and indicate if changes were made. 
Definition 1 A matrix $A$ is said to be an orthogonal projection matrix if it is idempotent $\left(A^{2}=A\right)$ and symmetric $\left(A^{T}=A\right)$.

Definition 2 Suppose that an experiment is carried out according to an array $A=$ $\left(a_{i j}\right)_{n \times m}=\left(a_{1}, \ldots, a_{m}\right)$, and $Y=\left(Y_{1}, \ldots, Y_{n}\right)^{T}$ is the experimental data vector. In the analysis of variance $S_{j}^{2}$, the sum of squares of the $j$ th factor, is defined as

$$
S_{j}^{2}=\sum_{i=0}^{p_{j}-1} \frac{1}{\left|I_{i j}\right|}\left(\sum_{s \in I_{i j}} Y_{s}\right)^{2}-\frac{1}{n}\left(\sum_{s=1}^{n} Y_{s}\right)^{2},
$$

where $I_{i j}=\left\{s: a_{s j}=i\right\}$ and $\left|I_{i j}\right|$ is the number of elements in $I_{i j}$. From (1), $S_{j}^{2}$ is a quadratic form in $Y$ and there exists a unique symmetric matrix $A_{j}$ such that $S_{j}^{2}=Y^{T} A_{j} Y$. The matrix $A_{j}$ is called the matrix image (MI) of the $j$ th column $a_{j}$ of $A$, denoted by $m\left(a_{j}\right)=A_{j}$. The MI of a subarray of $A$ is defined as the sum of the MIs of all its columns. In particular, we denote the MI of $A$ by $m(A)$. Let $1_{r}$ be the $r \times 1$ vector of 1 's. Then $m\left(1_{r}\right)=P_{r}$ where $P_{r}=\frac{1}{r} 1_{r} 1_{r}^{T}$.

Let $(r)=(0, \ldots, r-1)^{T}, e_{i}(r)=\left(0 \cdots 0 i_{1}^{i} 0 \cdots 0\right)_{1 \times r}^{T}$ and $I_{r}$ be the identity matrix of order $r$. Then $m((r))=\tau_{r}$ where $\tau_{r}=I_{r}-P_{r}$. The following permutation matrices are very useful:

$$
N_{r}=e_{1}(r) e_{2}^{T}(r)+\cdots+e_{r-1}(r) e_{r}^{T}(r)+e_{r}(r) e_{1}^{T}(r)
$$

and

$$
K(p, q)=\sum_{i=1}^{p} \sum_{j=1}^{q} e_{i}(p) e_{j}^{T}(q) \otimes e_{j}(q) e_{i}^{T}(p),
$$

where $\otimes$ is the usual Kronecker product in the theory of matrices. Sometimes, it is necessary and easy to use the following properties of these two permutation matrices to obtain the orthogonal arrays needed:

$$
\begin{aligned}
& N_{r}(r)=1_{r}+(r), \quad \bmod r, \\
& K(p, q)\left(1_{q} \otimes(p)\right)=(p) \otimes 1_{q}, \quad K(p, q)((q) \otimes(p))=(p) \otimes(q)
\end{aligned}
$$

and

$$
K(p, q)\left(P_{q} \otimes \tau_{p}\right) K^{T}(p, q)=\tau_{p} \otimes P_{q}, \quad K(p, q)\left(\tau_{q} \otimes \tau_{p}\right) K^{T}(p, q)=\tau_{p} \otimes \tau_{q}
$$

Lemma 1 For any permutation matrix $S$ and any array $L, m\left(S\left(L \otimes 1_{r}\right)\right)=S\left(m(L) \otimes P_{r}\right) S^{T}$, and $m\left(S\left(1_{r} \otimes L\right)\right)=S\left(P_{r} \otimes m(L)\right) S^{T}$.

Lemma 2 Let $A$ be an $O A$ of strength 1, i.e.,

$$
A=\left(a_{1}, \ldots, a_{m}\right)=\left(S_{1}\left(1_{r_{1}} \otimes\left(p_{1}\right)\right), \ldots, S_{m}\left(1_{r_{m}} \otimes\left(p_{m}\right)\right)\right),
$$

where $r_{j} p_{j}=n$ and $S_{i}$ is a permutation matrix, for $i=1, \ldots, m$. The following statements are equivalent. 
(1) $A$ is an $O A$ of strength 2.

(2) $m(A)$ is a projection matrix.

(3) $m\left(a_{i}\right) m\left(a_{j}\right)=0(i \neq j)$.

(4) The projection matrix $\tau_{n}$ can be decomposed as $\tau_{n}=m\left(a_{1}\right)+\cdots+m\left(a_{m}\right)+\Delta$, where $\operatorname{rk}(\triangle)=n-1-\sum_{j=1}^{m}\left(p_{j}-1\right)$ is the rank of the matrix $\triangle$.

Lemma 3 Suppose $L$ and $H$ are OAs satisfying $m(L) m(H)=0$. Then $K=(L, H)$ is also an $O A$.

Lemma 4 Let $(L, H)$ and $K$ be $O A$ s of run size $n$. Then $(K, H)$ is also an $O A$ if $m(K) \leq m(L)$, where $A \leq B$ means that the difference $B-A$ is nonnegative.

Lemmas 1, 2, 3, and 4 can be found in Zhang et al. [3].

Now we state the following theorems.

Theorem 1 If $L=\left[(r) \otimes 1_{p}, H\right]$ is an $O A$, then $m(H) \leq I_{r} \otimes \tau_{p}$. If $L=\left[1_{p} \otimes(r), K\right]$ is an $O A$, then $m(K) \leq \tau_{p} \otimes I_{r}$.

Proof From Lemma 2, we have $m(L)=\tau_{r} \otimes P_{p}+m(H)$. Since $m(L) \leq \tau_{r p}, \tau_{r p}=\tau_{r} \otimes P_{p}+$ $I_{r} \otimes \tau_{p}$ and $m\left((r) \otimes 1_{p}\right)=\tau_{r} \otimes P_{p}$, it follows that $m(H) \leq I_{r} \otimes \tau_{p}$.

Similarly, we can prove that $m(K) \leq \tau_{p} \otimes I_{r}$.

Corollary If $p$ is a prime and $D(r, m ; p)$ is a p-level difference matrix, then both $D(r, m ; p) \oplus$ $(p)$ and $(p) \oplus D(r, m ; p)$ are $O A s$, and $m(D(r, m ; p) \oplus(p)) \leq I_{r} \otimes \tau_{p}$ and $m((p) \oplus D(r, m ; p)) \leq$ $\tau_{p} \otimes I_{r}$.

Proof From Bose and Bush [11], we see that

$$
L=\left[(r) \otimes 1_{p}, D(r, m ; p) \oplus(p)\right]
$$

is an OA. From Theorem 1, it follows that $m(D(r, m ; p) \oplus(p)) \leq I_{r} \otimes \tau_{p}$. Similarly, we can prove that $(p) \oplus D(r, m ; p)$ is an OA and $m((p) \oplus D(r, m ; p)) \leq \tau_{p} \otimes I_{r}$.

Theorem 2 If $p$ is a prime and $D(r, m ; p)$ is a p-level difference matrix, then $D(r, m ; p) \oplus$ $0_{q} \oplus(p)$ is an $O A$, and $m\left(D(r, m ; p) \oplus 0_{q} \oplus(p)\right) \leq I_{r} \otimes P_{q} \otimes \tau_{p}$.

Proof From Lemma 1 and Theorem 1, we have $m\left(D(r, m ; p) \oplus(p) \oplus 0_{q}\right) \leq I_{r} \otimes \tau_{p} \otimes P_{q}$,

$$
\begin{aligned}
m & \left(D(r, m ; p) \oplus 0_{q} \oplus(p)\right) \\
& =m\left(\left(I_{r} \otimes K(q, p)\right)\left(D(r, m ; p) \oplus(p) \oplus 0_{q}\right)\right) \\
& \leq\left(I_{r} \otimes K(q, p)\right)\left(I_{r} \otimes \tau_{p} \otimes P_{q}\right)\left(I_{r} \otimes K(q, p)\right)^{T} \\
& =I_{r} \otimes P_{q} \otimes \tau_{p} .
\end{aligned}
$$

Theorem 3 If $\tau_{p} \otimes \tau_{q}=\sum_{i=1}^{k} S_{i}\left(\tau_{p} \otimes P_{q}\right) S_{i}^{T}$ is an orthogonal decomposition of $\tau_{p} \otimes \tau_{q}$, then $I_{r} \otimes \tau_{p} \otimes \tau_{q}=\sum_{i=1}^{k}\left(I_{r} \otimes S_{i}\right)\left(I_{r} \otimes \tau_{p} \otimes P_{q}\right)\left(I_{r} \otimes S_{i}\right)^{T}$ is an orthogonal decomposition of $I_{r} \otimes \tau_{p} \otimes \tau_{q}$. If there exists an $O A H$ such that $m(H) \leq I_{r} \otimes \tau_{p}$, then $L=\left[\left(I_{r} \otimes S_{1}\right)(H \otimes\right.$ $\left.\left.1_{q}\right), \ldots,\left(I_{r} \otimes S_{k}\right)\left(H \otimes 1_{q}\right)\right]$ is also an $O A$. 
Proof Since $\tau_{p} \otimes \tau_{q}=\sum_{i=1}^{k} S_{i}\left(\tau_{p} \otimes P_{q}\right) S_{i}^{T}$, we have

$$
\begin{aligned}
I_{r} \otimes \tau_{p} \otimes \tau_{q} & =I_{r} \otimes\left(\sum_{i=1}^{k} S_{i}\left(\tau_{p} \otimes P_{q}\right) S_{i}^{T}\right) \\
& =\sum_{i=1}^{k}\left(I_{r} I_{r} I_{r}\right) \otimes\left(S_{i}\left(\tau_{p} \otimes P_{q}\right) S_{i}^{T}\right) .
\end{aligned}
$$

Using the property $(A B C) \otimes(D E F)=(A \otimes D)(B \otimes E)(C \otimes F)$, we obtain

$$
I_{r} \otimes \tau_{p} \otimes \tau_{q}=\sum_{i=1}^{k}\left(I_{r} \otimes S_{i}\right)\left(I_{r} \otimes \tau_{p} \otimes P_{q}\right)\left(I_{r} \otimes S_{i}\right)^{T}
$$

Because of the orthogonality in each step, the above decomposition of $I_{r} \otimes \tau_{p} \otimes \tau_{q}$ is orthogonal. By Lemma 1, we have

$$
m\left(\left(I_{r} \otimes S_{i}\right)\left(H \otimes 1_{q}\right)\right)=\left(I_{r} \otimes S_{i}\right)\left(H \otimes 1_{q}\right)\left(I_{r} \otimes S_{i}\right)^{T} \leq\left(I_{r} \otimes S_{i}\right)\left(I_{r} \otimes \tau_{p} \otimes P_{q}\right)\left(I_{r} \otimes S_{i}\right)^{T} .
$$

By Lemmas 3 and 4 , we see that $\left(I_{r} \otimes S_{i}\right)\left(H \otimes 1_{q}\right)$ and $\left(I_{r} \otimes S_{j}\right)\left(H \otimes 1_{q}\right)$ is orthogonal $(i \neq j)$. It follows from Lemma 2 that $L$ is an OA.

\section{Some examples}

These matrix inequalities in Theorems 1, 2, and 3 are very useful for construction of orthogonal arrays. We illustrate their applications with some examples. We begin with OAs $L_{9}\left(3^{4}\right)$ and $L_{16}\left(4^{5}\right)$ and their properties:

$$
L_{9}\left(3^{4}\right)=\left((3) \otimes 1_{3}, 1_{3} \otimes(3), a, b\right)=\left[\begin{array}{ccccccccc}
0 & 0 & 0 & 1 & 1 & 1 & 2 & 2 & 2 \\
0 & 1 & 2 & 0 & 1 & 2 & 0 & 1 & 2 \\
0 & 1 & 2 & 1 & 2 & 0 & 2 & 0 & 1 \\
0 & 1 & 2 & 2 & 0 & 1 & 1 & 2 & 0
\end{array}\right]^{T} .
$$

For this OA $L_{9}\left(3^{4}\right)$, there exists a $9 \times 9$ permutation $T_{2}$ (as follows) such that $\left((3) \otimes 1_{3}, 1_{3} \otimes\right.$ $(3))=T_{2}(a, b)$. From Lemma 2 , we have $m\left(L_{9}\left(3^{4}\right)\right)=\tau_{9}, m(a, b)=\tau_{9}-\tau_{3} \otimes P_{3}-P_{3} \otimes \tau_{3}=$ $\tau_{3} \otimes \tau_{3}$ and $m\left((3) \otimes 1_{3}, 1_{3} \otimes(3)\right)=T_{2}\left(\tau_{3} \otimes \tau_{3}\right) T_{2}^{T}$. Hence

$$
\tau_{9}=\sum_{i=1}^{2} T_{i}\left(\tau_{3} \otimes \tau_{3}\right) T_{i}^{T}
$$

where $T_{1}=I_{9}$ and

$$
T_{2}=\left(\begin{array}{lllllllll}
1 & 0 & 0 & 0 & 0 & 0 & 0 & 0 & 0 \\
0 & 0 & 0 & 0 & 0 & 1 & 0 & 0 & 0 \\
0 & 0 & 0 & 0 & 0 & 0 & 0 & 1 & 0 \\
0 & 0 & 0 & 0 & 0 & 0 & 0 & 0 & 1 \\
0 & 1 & 0 & 0 & 0 & 0 & 0 & 0 & 0 \\
0 & 0 & 0 & 1 & 0 & 0 & 0 & 0 & 0 \\
0 & 0 & 0 & 0 & 1 & 0 & 0 & 0 & 0 \\
0 & 0 & 0 & 0 & 0 & 0 & 1 & 0 & 0 \\
0 & 0 & 1 & 0 & 0 & 0 & 0 & 0 & 0
\end{array}\right) .
$$


On the other hand, we have $\tau_{9}-\tau_{3} \otimes P_{3}-P_{3} \otimes \tau_{3}=\tau_{3} \otimes \tau_{3}$. Also, $\tau_{3} \otimes \tau_{3}=\sum_{i=1}^{2} Q_{i}\left(\tau_{3} \otimes\right.$ $\left.P_{3}\right) Q_{i}^{T}$, where $Q_{1}=\operatorname{diag}\left(I_{3}, N_{3}, N_{3}^{2}\right) K(3,3)$ and $Q_{2}=\operatorname{diag}\left(I_{3}, N_{3}^{2}, N_{3}\right) K(3,3)$.

Consider the orthogonal array $L_{16}\left(4^{5}\right)$ :

$$
\begin{aligned}
L_{16}\left(4^{5}\right) & =\left((4) \otimes 1_{4}, 1_{4} \otimes(4), c, d, f\right) \\
& =\left[\begin{array}{llllllllllllllll}
0 & 0 & 0 & 0 & 1 & 1 & 1 & 1 & 2 & 2 & 2 & 2 & 3 & 3 & 3 & 3 \\
0 & 1 & 2 & 3 & 0 & 1 & 2 & 3 & 0 & 1 & 2 & 3 & 0 & 1 & 2 & 3 \\
0 & 1 & 2 & 3 & 1 & 0 & 3 & 2 & 2 & 3 & 0 & 1 & 3 & 2 & 1 & 0 \\
0 & 1 & 2 & 3 & 2 & 3 & 0 & 1 & 3 & 2 & 1 & 0 & 1 & 0 & 3 & 2 \\
0 & 1 & 2 & 3 & 3 & 2 & 1 & 0 & 1 & 0 & 3 & 2 & 2 & 3 & 0 & 1
\end{array}\right]^{T} .
\end{aligned}
$$

From Lemma 2, we have $\tau_{16}=\sum_{i=1}^{2} S_{i}\left(\tau_{4} \otimes P_{4}\right) S_{i}^{T}+\tau_{4} \otimes \tau_{4}$, where $S_{1}=I_{16}$ and $S_{2}=K(4,4)$. Moreover, we have $\tau_{4} \otimes \tau_{4}=\sum_{i=3}^{5} S_{i}\left(\tau_{4} \otimes P_{4}\right) S_{i}^{T}$, where $S_{3}=\operatorname{diag}\left(Q_{1}^{\prime}, Q_{2}^{\prime}, Q_{3}^{\prime}, Q_{4}^{\prime}\right) K(4,4)$, $S_{4}=\operatorname{diag}\left(Q_{1}^{\prime}, Q_{3}^{\prime}, Q_{4}^{\prime}, Q_{2}^{\prime}\right) K(4,4), S_{5}=\operatorname{diag}\left(Q_{1}^{\prime}, Q_{4}^{\prime}, Q_{2}^{\prime}, Q_{3}^{\prime}\right) K(4,4), Q_{1}^{\prime}=I_{4}, Q_{2}^{\prime}=I_{2} \otimes N_{2}$, $Q_{3}^{\prime}=N_{2} \otimes I_{2}$ and $Q_{4}^{\prime}=N_{2} \otimes N_{2}$.

Example 1 (Construction of orthogonal arrays of run size 108) Orthogonally decompose the projection matrix $\tau_{108}$ as follows:

$$
\begin{aligned}
\tau_{108}= & I_{12} \otimes \tau_{9}+\tau_{12} \otimes P_{9} \\
= & I_{12} \otimes \tau_{3} \otimes \tau_{3}+I_{12} \otimes \tau_{3} \otimes P_{3}+I_{12} \otimes P_{3} \otimes \tau_{3} \\
& +\left(P_{6} \otimes \tau_{2}+P_{3} \otimes \tau_{2} \otimes \tau_{2}+\tau_{3} \otimes I_{4}+P_{3} \otimes \tau_{2} \otimes P_{2}\right) \otimes P_{9} \\
= & I_{12} \otimes \tau_{3} \otimes \tau_{3}+I_{12} \otimes \tau_{3} \otimes P_{3}+\left(P_{6} \otimes \tau_{2}+P_{3} \otimes \tau_{2} \otimes \tau_{2}\right) \otimes P_{9} \\
& +I_{12} \otimes P_{3} \otimes \tau_{3}+\left(\tau_{3} \otimes I_{4}+P_{3} \otimes \tau_{2} \otimes P_{2}\right) \otimes P_{9} \\
= & I_{12} \otimes \tau_{3} \otimes \tau_{3}+\left(I_{12} \otimes \tau_{3}+\left(P_{6} \otimes \tau_{2}+P_{3} \otimes \tau_{2} \otimes \tau_{2}\right) \otimes P_{3}\right) \otimes P_{3} \\
& +Q\left(\left(I_{12} \otimes \tau_{3}+\left(\tau_{3} \otimes I_{4}+P_{3} \otimes \tau_{2} \otimes P_{2}\right) \otimes P_{3}\right) \otimes P_{3}\right) Q^{T},
\end{aligned}
$$

where $Q=I_{12} \otimes K(3,3)$.

Now we want to find OAs $H_{1}, H_{2}$ and $H_{3}$ such that $m\left(H_{1}\right) \leq I_{12} \otimes \tau_{3} \otimes \tau_{3}$, and $m\left(H_{2}\right) \leq$ $I_{12} \otimes \tau_{3}+\left(P_{6} \otimes \tau_{2}+P_{3} \otimes \tau_{2} \otimes \tau_{2}\right) \otimes P_{3}$, and $m\left(H_{3}\right) \leq I_{12} \otimes \tau_{3}+\left(\tau_{3} \otimes I_{4}+P_{3} \otimes \tau_{2} \otimes P_{2}\right) \otimes P_{3}$.

Let $\oplus$ be the Kronecker sum $(\bmod 3)$ in ordinary matrix theory and $D(12,12,3)$ be a difference matrix as follows:

$$
D(12,12,3)=\left(\begin{array}{llllllllllll}
0 & 0 & 0 & 1 & 1 & 0 & 0 & 1 & 0 & 2 & 2 & 0 \\
0 & 0 & 0 & 0 & 2 & 0 & 2 & 0 & 2 & 0 & 0 & 1 \\
0 & 0 & 1 & 0 & 0 & 2 & 1 & 2 & 0 & 0 & 1 & 0 \\
0 & 0 & 2 & 2 & 0 & 1 & 0 & 0 & 1 & 1 & 0 & 0 \\
0 & 1 & 2 & 2 & 0 & 0 & 1 & 1 & 2 & 0 & 2 & 2 \\
0 & 1 & 2 & 1 & 2 & 1 & 2 & 2 & 2 & 2 & 1 & 0 \\
0 & 1 & 0 & 0 & 2 & 2 & 0 & 2 & 1 & 1 & 2 & 2 \\
0 & 1 & 1 & 2 & 1 & 2 & 2 & 0 & 0 & 2 & 0 & 2 \\
0 & 2 & 1 & 2 & 1 & 0 & 0 & 2 & 2 & 1 & 1 & 1 \\
0 & 2 & 1 & 0 & 0 & 1 & 2 & 1 & 1 & 2 & 2 & 1 \\
0 & 2 & 2 & 1 & 2 & 2 & 1 & 1 & 0 & 1 & 0 & 1 \\
0 & 2 & 0 & 1 & 1 & 1 & 1 & 0 & 1 & 0 & 1 & 2
\end{array}\right) .
$$


It follows from the corollary of Theorem 1 that $H=L_{36}\left(3^{12}\right)=D(12,12,3) \oplus(3)$ is an OA and $m(H) \leq I_{12} \otimes \tau_{3}$. From the property $\tau_{3} \otimes \tau_{3}=\sum_{i=1}^{2} Q_{i}\left(\tau_{3} \otimes P_{3}\right) Q_{i}^{T}$ and Theorem 3, we have an orthogonal array $H_{1}$ :

$$
H_{1}=L_{108}\left(3^{24}\right)=\left[\left(I_{r} \otimes Q_{1}\right)\left(H \otimes 1_{3}\right),\left(I_{r} \otimes Q_{2}\right)\left(H \otimes 1_{3}\right)\right]
$$

and $m\left(H_{1}\right) \leq I_{12} \otimes \tau_{3} \otimes \tau_{3}$.

From the orthogonal array $L_{36}\left(6^{2} 3^{4} 2^{9}\right)$ in Zhang et al. [4], we can get an orthogonal array $L_{36}^{1}\left(6^{2} 3^{4} 2^{9}\right)=K(12,3) L_{36}\left(6^{2} 3^{4} 2^{9}\right)=\left[L_{12}\left(2^{9}\right) \otimes 1_{3}, L_{36}\left(6^{2} 3^{4}\right)\right]$ satisfying $m\left(L_{36}^{1}\left(6^{2} 3^{4} \times\right.\right.$ $\left.\left.2^{9}\right)\right) \leq \tau_{36}=I_{12} \otimes \tau_{3}+\tau_{12} \otimes P_{3}$. Deleting the orthogonal array $L_{12}\left(2^{9}\right) \otimes 1_{3}$ from $L_{36}^{1}\left(6^{2} 3^{4} 2^{9}\right)$, we obtain an OA $H_{2}=L_{36}\left(6^{2} 3^{4}\right)$ whose MI satisfies $m\left(H_{2}\right) \leq I_{12} \otimes \tau_{3}+\left(P_{6} \otimes \tau_{2}+P_{3} \otimes \tau_{2} \otimes\right.$ $\left.\tau_{2}\right) \otimes P_{3}$.

By the definition of an OA, there exists a permutation matrix $T$ such that $T L_{36}\left(6^{2} 3^{4} 2^{9}\right)$ contains the two columns $1_{6} \otimes(2) \otimes 1_{3}$ and $1_{3} \otimes((2) \oplus(2)) \otimes 1_{3}$. Deleting these two columns from $T L_{36}\left(6^{2} 3^{4} 2^{9}\right)$, we obtain an OA $H_{3}=L_{36}\left(6^{2} 3^{4} 2^{7}\right)$ satisfying $m\left(H_{3}\right) \leq I_{12} \otimes \tau_{3}+\left(\tau_{3} \otimes\right.$ $\left.I_{4}+P_{3} \otimes \tau_{2} \otimes P_{2}\right) \otimes P_{3}$.

Hence we can construct a new OA $L_{108}\left(6^{4} 3^{32} 2^{7}\right)$ as follows:

$$
L_{108}\left(6^{4} 3^{32} 2^{7}\right)=\left[H_{1}, H_{2} \otimes 1_{3}, Q\left(H_{3} \otimes 1_{3}\right)\right] .
$$

The percent saturation for this $\mathrm{OA}$ is $84.2 \%$.

Also, similarly constructing of $H_{2}$ and by use of the orthogonal arrays $L_{36}\left(3^{2} 2^{20}\right)$, $L_{36}\left(3^{3} 2^{13}\right), L_{36}\left(6^{1} 3^{2} 2^{11}\right)$ and $L_{36}\left(3^{1} 2^{27}\right)$ in Zhang et al. [3], $L_{36}\left(6^{1} 3^{1} 2^{18}\right)$ in Hedayet et al. [2], $L_{36}\left(3^{12} 2^{11}\right)$ and $L_{36}\left(6^{1} 3^{8} 2^{10}\right)$ in Zhang et al. [4], we can obtain seven OAs $L_{36}\left(3^{2} 2^{11}\right)$, $L_{36}\left(3^{1} 2^{18}\right), L_{36}\left(6^{1} 3^{2} 2^{2}\right), L_{36}\left(6^{1} 3^{1} 2^{9}\right), L_{36}\left(6^{2} 3^{4}\right), L_{36}\left(3^{12} 2^{2}\right)$ and $L_{36}\left(6^{1} 3^{8} 2^{1}\right)$ whose MIs are less than or equal to $I_{12} \otimes \tau_{3}+\left(P_{6} \otimes \tau_{2}+P_{3} \otimes \tau_{2} \otimes \tau_{2}\right) \otimes P_{3}$.

On the other hand, by the definition of an OA, any OA of run size 36 with two factors having two levels can contain the two columns $1_{6} \otimes(2) \otimes 1_{3}$ and $1_{3} \otimes((2) \oplus(2)) \otimes 1_{3}$ through row permutations. For example, there exists OA $L_{36}\left(9^{1} 3^{16}\right)$ in Example 2, $L_{36}\left(18^{1} 2^{2}\right)$, $L_{36}\left(3^{12} 2^{11}\right), L_{36}\left(2^{35}\right), L_{36}\left(3^{1} 2^{27}\right), L_{36}\left(6^{1} 3^{12} 2^{2}\right), L_{36}\left(3^{2} 2^{20}\right), L_{36}\left(3^{3} 2^{13}\right), L_{36}\left(6^{1} 3^{2} 2^{11}\right)$ in Zhang et al. [3], $L_{36}\left(6^{1} 3^{1} 2^{18}\right)$ in Hedayet et al. [2], $L_{36}\left(6^{1} 3^{8} 2^{10}\right), L_{36}\left(6^{2} 3^{9} 2^{3}\right), L_{36}\left(6^{2} 3^{5} 2^{2}\right), L_{36}\left(6^{3} 2^{8}\right)$ in Zhang et al. [4], and $L_{36}\left(6^{2} 3^{1} 2^{10}\right), L_{36}\left(6^{3} 3^{1} 2^{4}\right), L_{36}\left(6^{3} 3^{2} 2^{3}\right)$ in Xu [12]. Deleting these two columns $1_{6} \otimes(2) \otimes 1_{3}$ and $1_{3} \otimes((2) \oplus(2)) \otimes 1_{3}$ from these arrays, we can obtain 17 OAs $L_{36}\left(9^{1} 3^{14}\right), L_{36}\left(18^{1}\right), L_{36}\left(3^{12} 2^{9}\right), L_{36}\left(2^{33}\right), L_{36}\left(3^{1} 2^{25}\right), L_{36}\left(6^{1} 3^{12}\right), L_{36}\left(3^{2} 2^{18}\right), L_{36}\left(3^{3} 2^{11}\right)$, $L_{36}\left(6^{1} 3^{2} 2^{9}\right), \quad L_{36}\left(6^{1} 3^{1} 2^{16}\right), \quad L_{36}\left(6^{1} 3^{8} 2^{8}\right), \quad L_{36}\left(6^{2} 3^{9} 2^{1}\right), \quad L_{36}\left(6^{2} 3^{5}\right), \quad L_{36}\left(6^{3} 2^{6}\right)$, $L_{36}\left(6^{2} 3^{1} 2^{8}\right), L_{36}\left(6^{3} 3^{1} 2^{2}\right)$, and $L_{36}\left(6^{3} 3^{2} 2^{1}\right)$ whose MIs are less than or equal to $I_{12} \otimes \tau_{3}+$ $\left(\tau_{3} \otimes I_{4}+P_{3} \otimes \tau_{2} \otimes P_{2}\right) \otimes P_{3}$.

Replacing $H_{2}$ in (2) by those seven OAs and $H_{3}$ in (2) by these $17 \mathrm{OAs}$, respectively, we can construct $(7+1) \times(17+1)-1=143$ OAs such as $L_{108}\left(6^{5} 3^{28} 2^{6}\right)$, etc. The orthogonal arrays constructed not only are new but also have higher percent saturations.

Example 2 (Construction of orthogonal arrays of run size 144) From the above properties of OAs $L_{9}\left(3^{4}\right)$ and $L_{16}\left(4^{5}\right)$, we have

$$
\tau_{9}=\sum_{i=1}^{2} T_{i}\left(\tau_{3} \otimes \tau_{3}\right) T_{i}^{T},
$$


where $T_{1}=I_{9}$ and $T_{2}$ is the above permutation matrix and

$$
\tau_{16}=\sum_{i=1}^{2} S_{i}\left(\tau_{4} \otimes P_{4}\right) S_{i}^{T}+\tau_{4} \otimes \tau_{4} .
$$

By using the properties $I_{9}=I_{9} I_{9} I_{9}, T_{i} I_{9} T_{i}^{T}=I_{9}, S_{i} P_{16} S_{i}^{T}=P_{16}(i=1,2),(A B C) \otimes(D E F)=$ $(A \otimes D)(B \otimes E)(C \otimes F)$, we can orthogonally decompose $\tau_{144}$ as follows:

$$
\begin{aligned}
\tau_{144} & =I_{9} \otimes \tau_{16}+\tau_{9} \otimes P_{16} \\
& =\sum_{i=1}^{2}\left(T_{i} \otimes S_{i}\right)\left(\left(I_{9} \otimes \tau_{4}+\tau_{3} \otimes \tau_{3} \otimes P_{4}\right) \otimes P_{4}\right)\left(T_{i} \otimes S_{i}\right)^{T}+I_{9} \otimes \tau_{4} \otimes \tau_{4} .
\end{aligned}
$$

From the $L_{16}\left(4^{5}\right)$, we have

$$
\tau_{4} \otimes \tau_{4}=\sum_{i=3}^{5} S_{i}\left(\tau_{4} \otimes P_{4}\right) S_{i}^{T} .
$$

Hence, $\tau_{144}$ can be further decomposed as

$$
\begin{aligned}
\tau_{144}= & \sum_{i=1}^{2}\left(T_{i} \otimes S_{i}\right)\left(\left(I_{9} \otimes \tau_{4}+\tau_{3} \otimes \tau_{3} \otimes P_{4}\right) \otimes P_{4}\right)\left(T_{i} \otimes S_{i}\right)^{T} \\
& +\sum_{i=3}^{5}\left(I_{9} \otimes S_{i}\right)\left(I_{9} \otimes \tau_{4} \otimes P_{4}\right)\left(I_{9} \otimes S_{i}\right)^{T} .
\end{aligned}
$$

Now we want to find OAs $H_{1}$ and $H_{2}$ such that $m\left(H_{1}\right) \leq I_{9} \otimes \tau_{4}+\tau_{3} \otimes \tau_{3} \otimes P_{4}$ and $m\left(H_{2}\right) \leq I_{9} \otimes \tau_{4}$.

By the definition of an OA and through row permutation, any OA with two 3-level columns can contain the two columns $(3) \otimes 1_{12}, 1_{3} \otimes(3) \otimes 1_{4}$. For example, from the result in Bose and Bush [11], we see that $K_{1}=\left[1_{3} \otimes L_{12}(12),(3) \oplus D(12,12,3)\right]$ is an OA, where $D(12,12,3)$ is a difference matrix in Example 1.

It is clear that $K_{1}$ contains two columns $(3) \otimes 1_{12}$ and $(3) \oplus(3) \otimes 1_{4}$, and $\left(\operatorname{diag}\left(I_{3}, N_{3}^{2}, N_{3}\right) \otimes\right.$ $\left.I_{4}\right)\left[(3) \otimes 1_{12},(3) \oplus(3) \otimes 1_{4}\right]=\left[(3) \otimes 1_{12}, 1_{3} \otimes(3) \otimes 1_{4}\right]$.

Set $K_{2}=\left(\operatorname{diag}\left(I_{3}, N_{3}^{2}, N_{3}\right) \otimes I_{4}\right) K_{1}$, we get an OA $K_{2}=L_{36}\left(12^{1} 3^{12}\right)$, which contains two columns $(3) \otimes 1_{12}$ and $1_{3} \otimes(3) \otimes 1_{4}$. Deleting these two columns from $K_{2}$, we obtain an OA $H_{1}=L_{36}\left(12^{1} 3^{10}\right)$, whose MI satisfies $m\left(H_{1}\right)=\tau_{36}-\tau_{3} \otimes P_{12}-P_{3} \otimes \tau_{3} \otimes P_{4}=I_{9} \otimes \tau_{4}+$ $\tau_{3} \otimes \tau_{3} \otimes P_{4}$.

Now we need to find another OA $H_{2}$ such that $m\left(H_{2}\right) \leq I_{9} \otimes \tau_{4}$. By using row permutations of the orthogonal array $L_{36}\left(9^{1} 2^{16}\right)\left(c f\right.$. [13]), we can obtain an OA $L_{36}\left(9^{1} 2^{16}\right)$ as follows:

$$
\begin{aligned}
& L_{36}\left(9^{1} 2^{16}\right) \\
& \quad=\left[\begin{array}{ccccccccccccccccc}
0 & x & x & x & -y & x & y & x & -y & -y & -z & -y & y & x & -x & -z & y \\
1 & x & x & x & z & z & y & -x & x & z & -x & z & z & -x & -y & z & z \\
2 & x & -y & -x & y & y & y & -x & -x & x & -y & -y & z & z & -x & -x & y \\
3 & x & -z & y & x & -x & y & x & -y & z & y & y & x & -y & x & -x & -y \\
4 & x & x & -x & -y & -y & y & z & -x & y & -y & y & -x & y & x & x & z \\
5 & x & -x & z & -z & -x & y & x & y & y & y & -y & -y & -x & -x & x & y \\
6 & x & -x & -x & -x & x & y & z & x & -x & -y & x & -z & z & y & y & -z \\
7 & x & y & -y & z & -z & y & -z & y & -z & y & -x & y & z & -z & z & -y \\
8 & x & -x & x & y & -z & y & -x & z & -y & x & y & -y & x & x & -y & y
\end{array}\right],
\end{aligned}
$$


where $i=(i, i, i, i)^{T}$, for $i=0,1, \ldots, 8, x=(0,0,1,1)^{T},-x=(1,1,0,0)^{T}, y=(0,1,0,1)^{T},-y=$ $(1,0,1,0)^{T}, z=(0,1,1,0)^{T}$ and $-z=(1,0,0,1)^{T}$.

Deleting the column $(9) \otimes 1_{4}$ from the $L_{36}\left(9^{1} 2^{16}\right)$, we obtain an OA $H_{2}=L_{36}\left(2^{16}\right)$. By using Theorem 1, we see that $m\left(H_{2}\right) \leq I_{9} \otimes \tau_{4}$.

From Theorem 3 the decomposition of $\tau_{144}$, we construct a new OA $L_{144}\left(12^{2} 3^{20} 2^{48}\right)$ as follows:

$$
\begin{aligned}
L_{144} & \left(12^{2} 3^{20} 2^{48}\right) \\
= & {\left[\left(T_{1} \otimes S_{1}\right)\left(H_{1} \otimes 1_{4}\right),\left(T_{2} \otimes S_{2}\right)\left(H_{1} \otimes 1_{4}\right),\left(I_{9} \otimes S_{3}\right)\left(H_{2} \otimes 1_{4}\right),\right.} \\
& \left.\left(I_{9} \otimes S_{4}\right)\left(H_{2} \otimes 1_{4}\right),\left(I_{9} \otimes S_{5}\right)\left(H_{2} \otimes 1_{4}\right)\right] .
\end{aligned}
$$

The percent saturation for this OA is 76.4\%. In fact, the percent saturations for the arrays constructed by Wang and $\mathrm{Wu}$ [14] are between $49.7 \%$ and $53.8 \%$ and the highest percent saturation for the arrays with 144 runs constructed by Mandeli [10] is $62.2 \%$.

Also, by the definition of an OA, any OA of run size 36 with two factors having three levels can contain the two columns $(3) \otimes 1_{12}$ and $1_{3} \otimes(3) \otimes 1_{4}$ through row permutations. For example, there exist OAs $L_{36}\left(6^{2} 3^{8} 2^{1}\right), L_{36}\left(6^{1} 3^{2} 2^{11}\right), L_{36}\left(3^{2} 2^{20}\right)$ in Zhang et al. [3], $L_{36}\left(12^{1} 3^{12}\right), L_{36}\left(6^{1} 3^{12} 2^{2}\right), L_{36}\left(3^{13} 2^{4}\right), L_{36}\left(3^{12} 2^{11}\right), L_{36}\left(6^{2} 3^{5} 2^{2}\right), L_{36}\left(6^{1} 3^{8} 2^{10}\right), L_{36}\left(6^{2} 3^{4} 2^{9}\right)$, $L_{36}\left(6^{1} 3^{9} 2^{3}\right)$ in Zhang et al. [4], $L_{36}\left(6^{3} 3^{2} 2^{3}\right), L_{36}\left(6^{3} 3^{3} 2^{1}\right)$ in $\mathrm{Xu}$ [12], $L_{36}\left(6^{3} 3^{7}\right)$ in Finney [15], etc. having $(3) \otimes 1_{12}$ and $1_{3} \otimes(3) \otimes 1_{4}$. Deleting these two columns, we can obtain 14 OAs $L_{36}\left(6^{3} 3^{5}\right), L_{36}\left(6^{2} 3^{6} 2^{1}\right), L_{36}\left(6^{1} 2^{11}\right), L_{36}\left(6^{1} 3^{10} 2^{2}\right)$, and so on. Replacing $H_{1}$ in (3) by these OAs, respectively, we can construct 195 OAs such as $L_{144}\left(6^{6} 3^{10} 2^{48}\right)$, etc. The orthogonal arrays constructed not only are new but also have higher percent saturations.

\section{Competing interests}

The authors declare that they have no competing interests.

\section{Authors' contributions}

The research and writing of this manuscript was a collaborative effort of all the authors. All authors read and approved the final manuscript.

\section{Acknowledgements}

The authors sincerely thank the referees for their many valuable comments which help us improving the paper. This work is supported by the National Natural Science Foundation of China (Grant No. 11171093) and IRTSTHN (14IRTSTHN023).

Received: 5 January 2015 Accepted: 20 July 2015 Published online: 01 August 2015

\section{References}

1. Hedayet, AS, Pu, K, Stufken, J: On the construction of asymmetrical orthogonal arrays. Ann. Stat. 20, 2142-2152 (1992)

2. Hedayat, AS, Sloane, NJA, Stufken, J: Orthogonal Arrays: Theory and Applications. Springer, New York (1999)

3. Zhang, Y, Lu, Y, Pang, S: Orthogonal arrays obtained by orthogonal decomposition of projection matrices. Stat. Sin. 9(2), 595-604 (1999)

4. Zhang, Y, Pang, S, Wang, Y: Orthogonal arrays obtained by the generalized Hadamard product. Discrete Math. 238, 151-170 (2001)

5. Pang, S, Zhang, Y, Liu, S: Normal mixed difference matrix and the construction of orthogonal arrays. Stat. Probab. Lett. 69, 431-437 (2004)

6. Pang, S: Construction of a new class of orthogonal arrays. J. Syst. Sci. Complex. 20(3), 429-436 (2007)

7. Zhang, Y, Li, W, Mao, S, Zheng, Z: Orthogonal arrays obtained by generalized difference matrices with $g$ levels. Sci. China Math. 54(1), 133-143 (2011)

8. Chen, G, Ji, L, Lei, J: The existence of mixed orthogonal arrays with four and five factors of strength two. J. Comb. Des. 22(8), 323-342 (2014)

9. Du, J, Wen, Q, Zhang, J, Liao, X: New construction and counting of systemic orthogonal array of strength $t$. IEICE Trans. Fundam. Electron. Commun. Comput. Sci. E96-A(9), 1901-1904 (2013)

10. Mandeli, JP: Construction of asymmetrical orthogonal arrays having factors with a large non-prime power number of levels. J. Stat. Plan. Inference 47, 377-391 (1995)

11. Bose, RC, Bush, KA: Orthogonal arrays of strength two and three. Ann. Math. Stat. 23, 508-524 (1952) 
12. $\mathrm{Xu}, \mathrm{H}$ : An algorithm for constructing orthogonal and nearly orthogonal arrays with mixed levels and small runs. Technometrics 44, 356-368 (2002)

13. Kuhfeld, W: Orthogonal arrays (2015). http://support.sas.com/techsup/technote/ts723.html

14. Wang, J, Wu, CFJ: An approach to the construction of asymmetrical orthogonal arrays. J. Am. Stat. Assoc. 6, 450-456 (1991)

15. Finney, DJ: Some enumerations for the $6 \times 6$ Latin squares. Util. Math. 21, 137-153 (1982)

Submit your manuscript to a SpringerOpen ${ }^{\circ}$ journal and benefit from:

- Convenient online submission

- Rigorous peer review

- Immediate publication on acceptance

- Open access: articles freely available online

- High visibility within the field

- Retaining the copyright to your article 